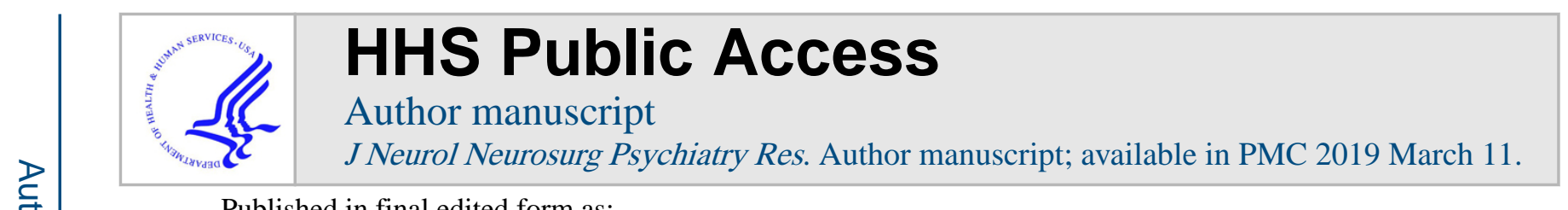

Published in final edited form as:

J Neurol Neurosurg Psychiatry Res. 2019 ; 1(1): .

\title{
A Pilot Study Validating Video-Based Training on Pre-Hospital Stroke Recognition
}

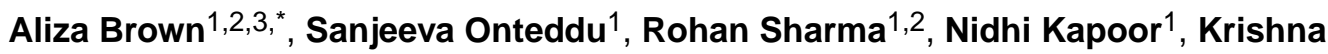 \\ Nalleballe ${ }^{1}$, Appathurai Balamurugan ${ }^{4}$, Sukumar Gundapaneni ${ }^{2}$, Nicolas Bianchi ${ }^{5}$, Robert \\ Skinner ${ }^{2}$, and William Culp ${ }^{1,2}$ \\ ${ }^{1}$ Department of Neurology, College of Public Health, University of Arkansas for Medical Sciences, \\ Little Rock, AR \\ 2Department of Radiology, College of Public Health, University of Arkansas for Medical Sciences, \\ Little Rock, AR \\ ${ }^{3}$ Department of Health Policy and Management, College of Public Health, University of Arkansas \\ for Medical Sciences, Little Rock, AR \\ ${ }^{4}$ Department of Chronic Disease Prevention and Control Branch, Arkansas Department of Health, \\ Little Rock, AR \\ ${ }^{5}$ Department of Neurology, Emory University, Atlanta, GA
}

\section{Abstract}

Introduction: Delays in recognizing stroke during pre-hospital emergency medical system (EMS) care may affect triage and transport time to an appropriate stroke ready hospital and may preclude patients from receiving time dependent treatment. All EMS transports in a large urban area in the stroke belt were evaluated for transport destinations, triage and transport time and stroke recognition following distribution ofan educational training video to local EMS services.

Hypothesis: Following video training, local paramedics will improve stroke recognition and shorten triage and transport time to appropriate stroke centers of care.

Methods: A training module ( $<10 \mathrm{~min})$ containing a stroke triage scenario, instruction on the Cincinnati Prehospital Stroke Score (CPSS) and the Los Angeles Prehospital Stroke Score (LAPSS) and 'where to transport' stroke patients was distributed and viewed by 96 paramedics. Data was collected from February to October 2016. Stroke recognition was determined from one primary stroke center (PSC) hospital's confirmation of EMS delivered patients (Site A). Yearly stroke recognition percentages of $44 \%$ from Site A in 2014 were used as baseline.

This article is distributed under the terms of the Creative Commons Attribution 4.0 International License (http://creativecommons.org/ licenses/by/4.0/), which permits unrestricted use, distribution, and reproduction in any medium, provided you give appropriate credit to the original author(s) and the source, provide a link to the Creative Commons license, and indicate if changes were made.

*Corresponding author: Aliza T. Brown, Department of Neurology College of Public Health, University of Arkansas for Medical Sciences, Arkansas, USA, brownalizat@uams.edu.

Conflict of Interest

None declared. 
Results: A total of 34,833 emergency 911 response transports were made with a total of 502 (1.4\%) suspected strokes identified by paramedics. Median [IQR] triage and transport time for stroke transports was 33 [2741] min. The PSC hospitals received a 5\% increase in stroke transports and non-specific care facilities decreased by 7\%. From 8,554 transports to site A (PSC) confirmed strokes totalled 107 transports with 139 suspected strokes by paramedics. Of these transports, 60 were correctly identified by paramedics (positive predictive value of $43 \%$, sensitivity of $56 \%$ ). By the second month following training, recognition percentages increased from baseline to $64 \%$. At five months, percentages of correct stroke identification had dropped to $36 \%$.

Conclusion: Video based training improved stroke recognition by an additional 19\%, but continual monthly or quarterly training is recommended for maintenance of increased stroke recognition.

\section{Keywords}

Stroke; Pre-hospital; EMS

\section{Introduction}

Stroke is the third leading cause of mortality in the world [1]. It is also the leading cause of chronic disability in the United States (U.S) [2]. The outcome of an acute ischemic stroke depends extensively on early diagnosis and reperfusion, usually by administration of tissue plasminogen activator (tPA) [3,4]. It has been estimated that for every min that stroke is untreated, a typical patient loses about 1.9 million neurons [5]. Since the current American Stroke Association guidelines for tPA eligibility is within 4.5 hours, time is a major limiting factor for tPA administration [6,7]. Several factors can cause delay in tPA administration including delay by the patients' families in calling 911 emergency medical services (EMS), lack of training by EMS and hospital emergency department staff, delay in obtaining radiological imaging and interpretation, and physicians' uncertainty about administering thrombolytics [8-10].

Pre-hospital notification by emergency medical services (EMS) has been associated with improved clinical assessment and higher rates of tPA delivery with reduced door- to-needle times in the hospital $[11,12]$. These in turn have been found to improve stroke outcomes $[13,14]$. Several pre-hospital stroke assessment models have been developed for stroke screening by the EMS responders including shortened Cincinnati Prehospital Stroke Scale (CPSS), Los Angeles Motor Scale, NIH Stroke scale (NIHSS), Medic Prehospital Assessment for Code Stroke (Med PACS) and others. These assessment models have shown modest improvements in prehospital screening of stroke patients in previous studies [15-18]. We developed a video training module for pre-hospital screening and stroke recognition and distributed it to area EMS for testing.

Here we present the results over the nine-month study period to determine the training module's efficacy. 


\section{Methods}

A review of de-identified data collected by three area Emergency Medical Systems Agencies in Central Arkansas, the Arkansas Stroke Registry (a Get-With-the-Guidelines registered program) from the Arkansas Health Department (ADH) and the University of Arkansas for Medical Sciences stroke program (Site A) was conducted. All data encompasses a review of de-identified transport data and hospital stroke confirmations from February 1, 2016 to October 31, 2016. The study was approved by the local Institutional Review Board as not human subject research.

A video utilizing professional stroke actors, paramedics and an ambulance was made to simulate a real event scenario of a 911 call for stroke. During the call and throughout the simulations of examination, a stroke educator registered nurse discussed the need to decrease time to delivery and treatment, precise methods for triage and care provided in the ambulance. Methodology for both the Los Angeles Pre-Hospital Stroke Screen (LAPSS) and the Cincinnati Pre-Hospital Stroke Screen (CPSS) were presented and discussed. Also detailed during the video was the need for early notification to alert the hospital emergency department for arrival. A directional delivery map was presented during the video illustrating the location and names of the test county's primary stroke centers (PSC) and the one acute stroke ready hospital (ASRH). There were no comments made on the use of lights and sirens (L\&S) during transport. The ADH disseminated the video link and a survey. An incentive gift card was offered to encourage video training and was given after completing an opinion survey regarding the video training.

To measure the impact of the video training on correct paramedic identification and management of 'suspected' strokes all transport data during the study period was collected for nine months following video release and survey return and then compared to pre-video release data that was collected a year prior to determine baseline stroke recognition rates and destination for stroke suspect transports according to hospital qualification in stroke care (non-specified care facilities (NSCF), ASRH or PSC) during the same months.

To determine the number of 'positive' and 'missed' strokes by the paramedics, all 911 ground response EMS transports delivering to one PSC (Site A) were matched against hospital ED records of strokes. Transport data used to match hospital ED cases of stroke included de-identified EMS information such as date, ER 'seen time', patient race, gender and age. Paramedic experience was taken into account as Years of Service (YOS) which was collected and categorized into 5 groups: < 1 year, 1 to 5 years, 5 to 10 years, 10 to 15 years and over 15 years of experience. The deidentified paramedic variables of years of service and reported suspected cases of stroke were evaluated based on correct and incorrect recognition.

The paramedic's baseline percentage of correct stroke identification was determined in a previous study which indicated that positive stroke recognition percentages were $44 \%$. This percentage was used as a comparison for the subsequent months of positive percentages of stroke recognition. 
Triage time and transport time from the scene to hospital destination were obtained for all paramedic suspected stroke transports during the study period. Both time epochs were calculated as one 'transport time' in min [IQR] and compared by distance travelled and travel mode (L\&S or none) during the study period.

We calculated diagnostic sensitivity, specificity, accuracy and related measures across the different groups of paramedics YOS and overall sensitivity across time before and after video training.

\section{Results}

\section{Video Training:}

A total of 96 paramedics underwent the training with a $100 \%$ rate of reporting compliance. A total of 70 paramedics were from the test county with another 26 from surrounding areas. The survey results indicated that $9 \%$ of the paramedics had not previously performed the CPSS and 4\% had not performed the LAPSS. Ninety-five percent of the paramedics thought the video would be helpful in demonstrating the use of both tests.

There were 34,833 EMS transports in the Test County during the study period (excluding 373 interfacility transports) out of which 502 (1.4\%) were suspected strokes. These 'stroke suspects' were either transported to the ASRH, NSCF, or to area PSCs at $\mathrm{n}=5(1 \%),=142$ (28\%) and $=355$ (71\%), respectively. The PSC hospitals received a 5\% increase in stroke transports and the NSCFs decreased by $7 \%$. Site A received a representative sample of 139 of suspected cases out of 8,554 total EMS transports (1.6\%) and took care of approximately 107 stroke patients.

Sixty paramedics transported suspected stroke patients to Site A during the study period. Their YOS are presented in Table 1 and is comparable to the paramedics YOS for all stroke transports. The paramedics with 5 to 10 YOS delivered the majority of stroke suspect transports at $41.7 \%$. Paramedics YOS for hospital verified stroke transports to Site A during the study period are presented in Table 2 .

The paramedics correctly identified stroke transports (Table 2) with a sensitivity of $56.1 \%$ [IQR 46.2-65.7] and a positive predictive value (PPV) was 43.1\% [IQR36.6-50], however their diagnostic accuracy was $98.5 \%$ [IQR 98.3-98.8] due to the low prevalence of the disease among all transports. The rate of true positive recognition by the month following video dissemination is shown in Figure 1. Recognition increased by 5\% during the month of training and an additional $14 \%$ by the second month but then gradually declined to baseline or lower at $35.7 \%$ within four months.

Overall combined triage and transport time for all stroke suspects was 33 min [IQR 27-41]. Data on median triage and transport time by vehicle territory is shown in Table 3.

The data indicates that transports inside the county area and metro areas had similar median times to destination hospitals. Some stroke transports used lights and sirens (L\&S) during commute and influenced transport time as shown in Figure 2. 
Vehicles originating from outside county areas (OCA) that used L\&S had shorter median delivery times vs. no L\&S (None) transports (56.6 [IQR 36.7-58] vs. 62.4 [IQR 47-69] $\min )$.

Transports within the county area (ICA) did not decrease delivery time with L\&S vs. None at 35.7 [IQR27.7-44] vs. 32.9 [IQR 29-43], respectively.

Those transports using L\&S in the metro county area (MCA) had an improvement vs. None at 25.3 [IQR 20.2-32] vs. 30 [IQR 25-35] min, respectively.

\section{Discussion}

Pre-hospital stroke assessment and notifications have been known to decrease the door to needle time, increase the rate of tPA delivery, and improve stroke outcomes [11-14]. Previous studies have found the diverse sensitivity of prehospital stroke assessment increase from $44 \%$ to as high as $91 \%$ [19-21]. Such varied results are due to differences in assessment scales, state population and location, urban versus rural settings, training level of paramedics, volume of strokes seen by paramedics and other factors such as trained emergency medical dispatchers (EMD).

Following stroke recognition training in this study, the dramatic 19\% increase from baseline in sensitivity (Figure 1) was seen within two months. Wojner-Alexandrov et al. [20] also observed similar results in their study. The video training offered in this study has the potential to benefit the entire state if adapted for county specific ASRH hospital locations considering the large telestroke network within the state [22].

The training may have also helped the paramedics in directing potential stroke patients to PSCs, which are better equipped at handling stroke cases. Although rates of endovascular interventions on these patients were not studied, this might be important to analyze in future studies. Rates of endovascular intervention could be compared to large vessel occlusion detection scores learned from video-based training in prehospital screening and triage. The use of L\&S added an additional variable on triage and transport time (Figure 2). The median transport time for the suspected strokes indicated an improvement in time reduction when L\&S were used by paramedics in the OCA territory although its use was not discussed in the video. Triage and transport time in the metro county study area (MCA) compared to rural in county areas (ICA) were consistent (Table 3 ) until the transport modes using L\&S were applied (Figure 2). The data here suggests the use of L\&S in heavy city traffic (MCA) and on the longer highway commutes (OCA) entering the county area (Figure 2). Although more data and more variables need to be analyzed to draw conclusions about the use of $L \& S$ in stroke transport in our region.

Falling stroke recognition rates (Table 2, Figure 1), suggests the need for retraining at regular intervals. Further decreases in stroke recognition rate may have included potential seasonal effects. However, our video training provides a cost effective and easily accessible retraining instrument for this purpose. Similar to this study, re-training after 5-6 months has been utilized in other studies examining training methods for prehospital stroke recognition, which recommended retraining at 3 to 6 months [23-25]. 
Since the overall percentage of stroke patients assessed by paramedics in our study was very low (1.4\%), the paramedics may be less adept at recognizing stroke cases as compared to areas yielding a higher stroke volume [23]. The video training did improve paramedic stroke recognition rates by $19 \%$ and may continue to improve with retraining.

\section{Acknowledgement}

This study was supported in part by the UAMS Foundation Fund to Cure Stroke, NIH/NIGMS IDeA Program COBRE grant P30 GM 110702, Translational Research Institute (TRI), grant UL1TR000039 through the NIH National Center for Research Resources and National Center for Advancing Translational Sciences and Dr. Feifei Wei for her exemplary assistance with statistics

\section{References}

1. Murray CJ, Lopez AD. Mortality by cause for eight regions of the world: Global Burden of Disease Study. Lancet 1997; 349: 1269-1276. [PubMed: 9142060]

2. Roger VL. Heart disease and stroke statistics 2011 update: A report from the American Heart Association. Circulation 2011; 123: e18-e209. [PubMed: 21160056]

3. Mitka M Early treatment of ischemic stroke with intravenous tPA reduces disability risk. JAMA 2013;310:1111. [PubMed: 24045728]

4. Saver JL. Time to treatment with intravenous tissue plasminogen activator and outcome from acute ischemic stroke. JAMA 2013; 309: 24802488. [PubMed: 23780461]

5. Saver JL. Time is brain-quantified. Stroke 2006; 37: 263-266. [PubMed: 16339467]

6. Ahmed N Implementation and outcome of thrombolysis with alteplase 3-4.5 h after an acute stroke: An updated analysis from SITS-ISTR. Lancet Neurol 2010; 9: 866-874. [PubMed: 20667790]

7. Hacke W Thrombolysis with alteplase 3 to $4.5 \mathrm{~h}$ after acute ischemic stroke. N Engl J Med 2008; 359: 1317-1329. [PubMed: 18815396]

8. Bunch ME, Nunziato EC, Labovitz DL. Barriers to the use of intravenous tissue plasminogen activator for in-hospital strokes. J Stroke Cerebrovasc Dis 2012; 21: 808-811. [PubMed: 21640608]

9. Johnson M, Bakas T. A review of barriers to thrombolytic therapy: Implications for nursing care in the emergency department. J Neurosci Nurs 2010; 42: 88-94. [PubMed: 20422794]

10. Kwan J, Hand P, Sandercock P. A systematic review of barriers to delivery of thrombolysis for acute stroke. Age Ageing 2004; 33: 116-121. [PubMed: 14960425]

11. Bae HJ. Prehospital notification from the emergency medical service reduces the transfer and intrahospital processing times for acute stroke patients. J Clin Neurol 2010; 6: 138-142. [PubMed: 20944814]

12. Abdullah AR. Advance hospital notification by EMS in acute stroke is associated with shorter door-to-computed tomography time and increased likelihood of administration of tissueplasminogen activator. Prehosp Emerg Care 2008; 12: 426-431. [PubMed: 18924004]

13. Fonarow GC. Improving door-to-needle times in acute ischemic stroke: The design and rationale for the American Heart Association/American Stroke Association's Target: Stroke initiative. Stroke 2011; 42: 2983-2989. [PubMed: 21885841]

14. Lin CB. Emergency medical service hospital prenotification is associated with improved evaluation and treatment of acute ischemic stroke. Circ Cardiovasc Qual Outcomes 2012; 5: 514-22. [PubMed: 22787065]

15. Kim JT. Field validation of the Los Angeles motor scale as a tool for paramedic assessment of stroke severity. Stroke 2017; 48: 298-306. [PubMed: 28087807]

16. Studnek JR. Assessing the validity of the Cincinnati prehospital stroke scale and the medic prehospital assessment for code stroke in an urban emergency medical services agency. Prehosp Emerg Care 2013; 17: 348-353. [PubMed: 23495755]

17. Purrucker JC. Design and validation of a clinical scale for prehospital stroke recognition, severity grading and prediction of large vessel occlusion: The shortened NIH Stroke Scale for emergency medical services. BMJ Open 2017; 7: e016893. 
18. You JS. Predictive value of the Cincinnati prehospital stroke scale for identifying thrombolytic candidates in acute ischemic stroke. Am J Emerg Med 2013 31(12): p. 1699-702. [PubMed: 24055479]

19. Kidwell CS. Identifying stroke in the field. Prospective validation of the Los Angeles prehospital stroke screen (LAPSS). Stroke 2000; 31: 71-76. [PubMed: 10625718]

20. Wojner-Alexandrov AW. Houston paramedic and emergency stroke treatment and outcomes study (HoPSTO). Stroke 2005; 36: 1512-1518. [PubMed: 15961712]

21. Ramanujam P Accuracy of stroke recognition by emergency medical dispatchers and paramedicsSan Diego experience. Prehosp Emerg Care 2008; 12: 307-313. [PubMed: 18584497]

22. Hsieh HC, Hsieh CY. Development of an educational program for staffs of emergency medical service to improve their awareness of stroke within $3 \mathrm{~h}$ of symptom onset: A pilot study. Acta Neurol Taiwan 2013; 22: 4-12. [PubMed: 23479240]

23. Ramanujam P, Guluma KZ. Accuracy of stroke recognition by emergency medical dispatchers and paramedics-San Diego Experience. Prehospital Emergency Care 2008; 12: 307-313. [PubMed: 18584497]

24. Bray JE, Martin J. An interventional study to improve paramedic diagnosis of stroke. Prehosp Emerg Care 2005; 9: 297-302. [PubMed: 16147479]

25. Nalleballe K Sharma R, Brown A, et al. Ideal telestroke time targets: Telestroke-based treatment times in the United States stroke belt. J Telemed Telecare. 2019 


\section{Timeto re-train?}

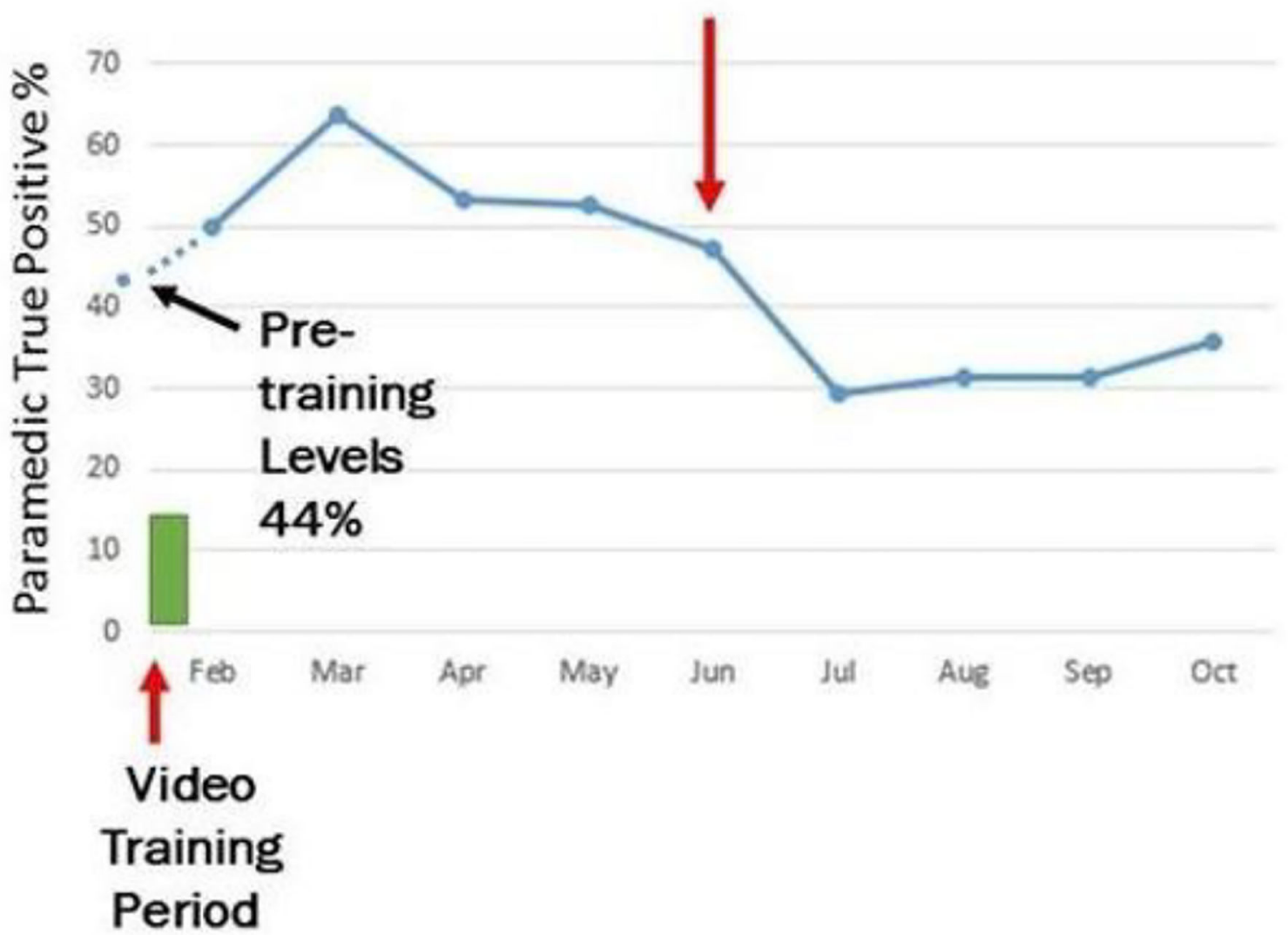

Figure 1:

Monthly percentages of pre-hospital stroke recognition by paramedics. Pre- training recognition rates of $44 \%$ increased by $19 \%$ in the second month following training. By the fifth month (July) the rates were declining to pre-training levels. 


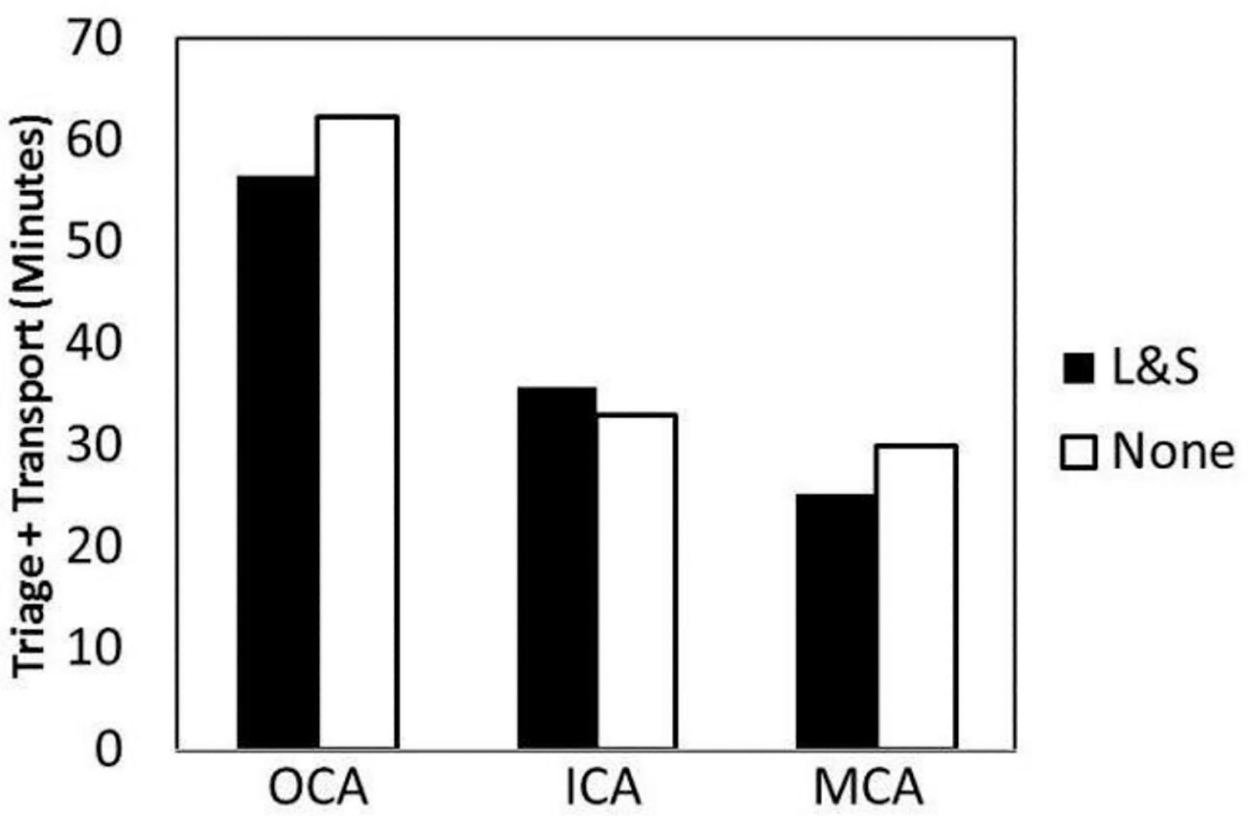

Figure 2:

Combining median triage and transport time and vehicle territory to hospital destination with the transport mode variable (Lights and Sirens [L\&S]). Vehicles utilizing lights and sirens (L\&S) decreased median triage and transport time to area hospitals in the study test county compared to no lights and sirens (None) from transports originating from outside county area (OCA) and in the metro county area (MCA) territories. The in-county area (ICA) stroke transports did not improve median triage and transport time using L\&S.

Median data represented. Black bars represent L\&S and None are shown by the white bars. 
Table 1:

Number of Paramedic's by Years of Service (YOS).

\begin{tabular}{|c|c|c|}
\hline Criteria & $\begin{array}{l}\text { Delivering to Site } \\
\text { A for Stroke } \\
\text { Recognition }\end{array}$ & $\begin{array}{c}\text { Delivering } \\
\text { to all } \\
\text { hospitals }\end{array}$ \\
\hline YOS & $\mathrm{n}(\%)$ & $\mathrm{n}(\%)$ \\
\hline$<1$ year & $7(11.7)$ & $9(9.1)$ \\
\hline $1<\mathrm{YOS}<5$ & $14(23.3)$ & $26(26.5)$ \\
\hline $5<\mathrm{YOS}<10$ & $25(41.7)$ & $36(36.7)$ \\
\hline $10<\mathrm{YOS}<15$ & $12(20.0)$ & $19(19.3)$ \\
\hline YOS $>15$ & $2(3.3)$ & $8(8.1)$ \\
\hline
\end{tabular}




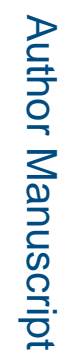

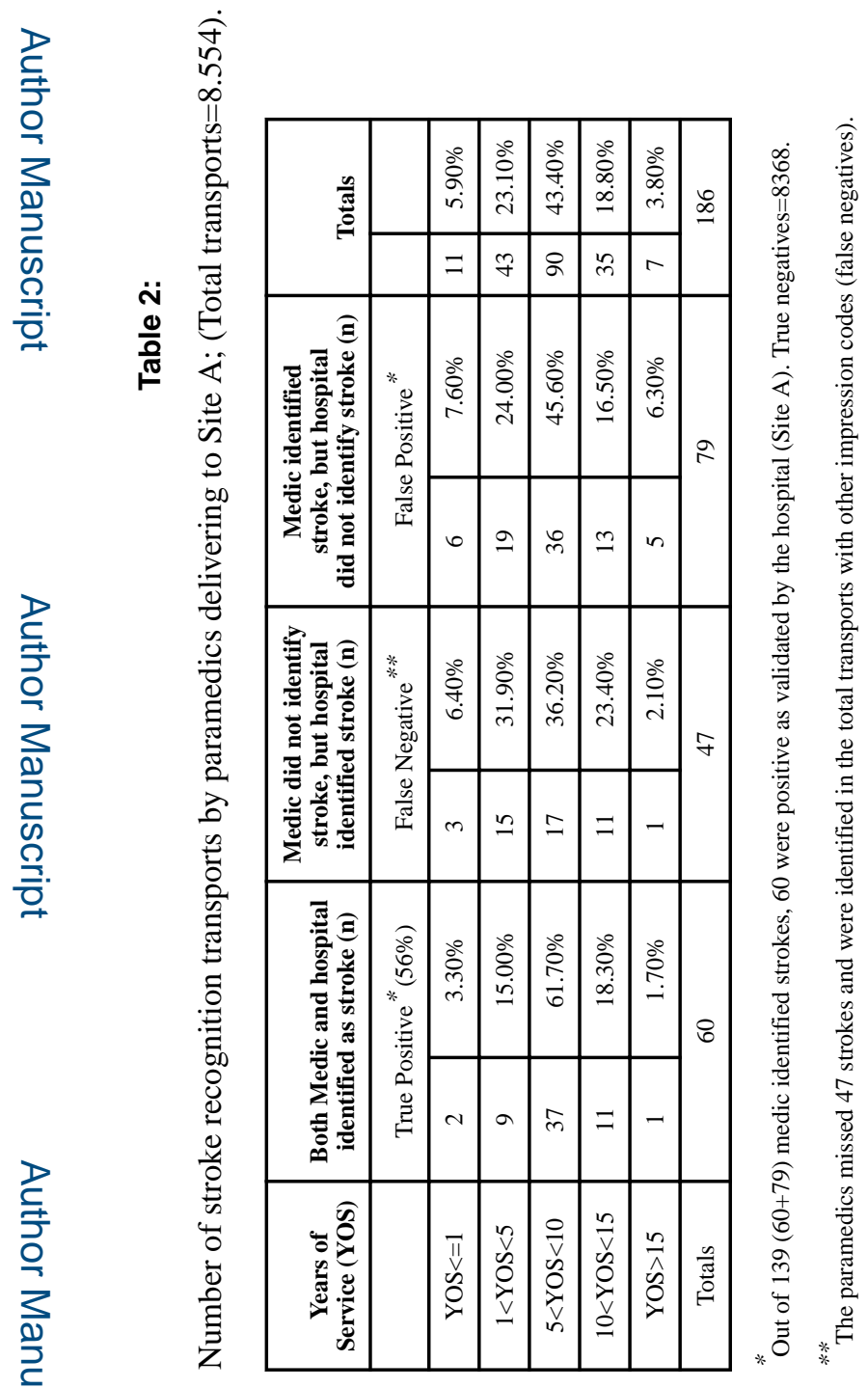


Table 3:

Median triage plus transport time of paramedic suspected strokes.

\begin{tabular}{|c|c|c|c|}
\hline $\begin{array}{c}\text { Vehicle } \\
\text { Territory }\end{array}$ & N & $\begin{array}{c}\text { Median } \\
\text { Time } \\
\text { (min) }\end{array}$ & [Q1-Q3] \\
\hline OCA & 11 & 58.4 & {$[44.1-65.7]$} \\
\hline ICA & 23 & 33.1 & {$[29.3-43.7]$} \\
\hline MCA & 105 & 29 & {$[23.7-34.5]$} \\
\hline
\end{tabular}

* OCA: Outside County Area; ICA: Inside County Area; MCA=In the Metro County Area 